\title{
Hypothetical inference and category structure
}

\author{
DEBORAH REDDING-STEWART and RUSSELL REVLIN \\ University of California, Santa Barbara, Santa Barbara, California 93106
}

\begin{abstract}
The present experiment considers whether the internal structure of statements plays a role in the way students reason about hypothetical domains. Students were asked to assign truth values to statements to form a consistent set of relations about hypothetical situations. The statements varied in degree of structure along two dimensions: across categories organization (generality) and within-categories organization (typicality). The results show that when generalities affirm a relation, they are readily accepted as defining a domain. The addition of within-categories structure makes only a marginal contribution to decisions. In contrast, there is a semantic structure effect for generalities that deny a relation. The findings accord with a two-stage process derived from a generality coding model.
\end{abstract}

Hypothetical inference occurs on those occasions when we conjecture about the ramification of situations whose truth status is either uncertain or false, but whose importance justifies further examination. A form of such reasoning called "prevarication" (Hockett, 1966) occurs when we assume, for the sake of argument, that a proposition is true, when, in fact, we know it is false. Revlis, Lipkin, and Hayes (1971) have introduced a task environment (borrowed from the philosophical literature: Rescher, 1964) to study hypothetical inference called "belief-contravening problems." The present research considers predictions from a model of hypothetical reasoning, the generality coding model (Revlis \& Hayes, 1972), by examining the importance of semantic factors for prevaricative judgments.

Belief-contravening problems are claimed to be paradigmatic of the kind of dilemmas individuals face when engaging in hypothetical reasoning, especially in those cases where they reason from assumptions they know to be false. The task environment consists of (1) a noncontradictory set of propositions, presumably representing relevant information for the judgments, and (2) a counterfactual assumption that introduces inconsistencies that the reasoner must resolve by assigning truth values to the propositions. For example: Propositions-(1) This mouse is a mammal; (2) all snakes are reptiles; (3) this mouse is not a snake. Assumption-This mouse is a snake. The reasoner is to accept the assumption and to assign a truth value of "false" to any statements that either individually or jointly lead to a logical contradiction when combined with the assumption. Revlis et al. (1971) found that people prefer to solve the problem by organizing the hypothetical universe of discourse with the most general statement in the set as the central element (e.g., by assigning Proposition 2 "true" and Proposition 1 "false"). Predictably, this preference for reasoning with generalities is more pronounced when people affirm a relation than when they deny one. Revlis and Hayes (1972) claim that this preference for generalities is a result of people's ability to structure the hypothetical universe of discourse in which they are embedded.

There are at least two ways in which generalities can provide structuring: (1) by the imposition of taskindependent organization associated with generalities in semantic memory, and (2) by offering a kind of taskdependent "inference ticket" (Ryle, 1949) that provides a link among potential categories (which may already be known to the reasoner or may be introduced in the hypothetical universe of discourse). While discussions by Revlis and his colleagues lend credence to the notion of an inference rule, the importance of semantic factors has yet to be explored. It is the purpose of the present study to examine the effect of semantic relations in providing the structuring for hypothetical problems.

Two conflicting findings on the effect of inferential context are pertinent here. First, semantic content per se is not a necessary component of hypothetical judgments. Reasoners assign generalities "true" even in those cases where the relations expressed are nonsensical or amphigorous (Revlis et al., 1971). Second, reasoners' judgments can reflect the available semantic structure of the relations reasoned about. Generalities that are believed to be true of the world external to the problem (not delimited in space and time) are treated as central elements in the problem solution more often than are generalities that are more restricted in their semantic range (arbitrary, delimited in space and time) (Revlis \& Hayes, 1972). These data show that when some external truth value is available to distinguish among assertions, reasoners may organize their judgments so that long-term memory components are imposed on the universe of discourse. When no such components are available, as in the problems with nonsense relations, the reasoners may rely solely on the ability of the generality to structure the domain by virtue of its intrinsic organizational properties (Revlis, 1974).

There are many possible semantic relations that may enter into the reasoner's judgments by way of structuring the hypothetical domain. One such semantic 
component is the organization inherent in the nature of the categories contained in the statements. The categories reasoned about have their own internal organization in addition to any imposed by the domain (Rosch, 1973). This category structure is reflected in the defining and characteristic exemplars of the categories. For example, "robin" is considered more closely associated with the category "bird" (i.e., more typical of the category) than, say, "ostrich." In a sense, both natural and artificial categories cohere as a unit where their exemplars are organized as a family of resemblances (Rosch \& Mervis, 1975).

If semantic relations do impart structure to hypothetical domains, why do they differentially enhance the acceptability of universally quantified relations more than particularly quantified ones? There does not appear to be any independent justification for assuming that generalities possess greater semantic structuring than particulars. Two possibilities immediately present themselves. It may be that the findings of Revlis et al. (1971) result from a confounding of semantic structure and the generality of the statements. Generalities may have been constructed to possess greater internal structure than the contrasted particulars. Alternatively, reasoners may focus primarily on the properties of the generalities and ignore those properties that are present only in the particulars. This may be expressed as a twostage process where, in the first stage, the reasoner attends to the generality and extracts features whose presence or absence is then examined in the particulars; components not present in the generalities are neither extracted nor examined. Second, only those semantic features found to discriminate between generalities and particulars are employed in the decision process. One prediction from this is that semantic elements will play a role in the decisions on problems where the generality possesses considerable semantic structure (highly typical relation), while the particular possesses little semantic structure (low typicality). In addition, this semantic structure effect will be seen most prominently among negative generalities whose ability to impart an organizational framework is normally minimal.

\section{METHOD}

The present experiment considers whether the internal structure of propositions plays a role in the truth-value assignments in hypothetical reasoning problems. Before considering the experimental manipulations, it will be useful to take a second look at the belief-contravening problem shown above. The reasoner is to assign a truth value of "false" to any statements that lead to a logical contradiction. In this problem, Proposition 3 is false because it directly contradicts the assumption. In addition, if the reasoner seeks to organize the domain around general rules, then the generality (Proposition 2) will be assigned "true" and the particular (Proposition 1), "false." This is required because the reasoner knows that the mouse is a snake (the assumption) and that all snakes are reptiles (Proposition 2); therefore, Proposition 1 must be false when it claims that the mouse is a mammal. An alternative solution is to organize the problem around a particular statement, in which case Proposition 1 is true and Proposition 2 is false. Although either solution path is equally logical, reasoners express a preference for organizing the problem around generalities.

\section{Subjects}

The subjects were 96 introductory psychology students who participated to fulfill a course requirement. The subjects were randomly assigned to one of four experimental conditions.

\section{Procedure}

Category structure in this study is synonymous with typicality (Rosch, 1973), which is operationalized as a relation where the superordinate category and the typical instance are coreferential: Given one, the reasoner will infer the other (see below).

Local norms were obtained in order to construct relations with uniformly high-typicality instances. This was accomplished in the following manner. First, category norms for seven categories (bird, fish, reptile, insect, flower, tree, and fruit) were collected from 80 students in an undergraduate psychology course. The students were randomly divided into two groups of 40 with each group given a separate questionnaire. The questionnaires instructed the students to respond in either of the following ways: (1) Given a category, the students were asked to supply instances for that category, or (2) given an instance, the students were asked to supply the category for that instance. Two prototypical instances were chosen for each category such that, given the category, these two instances were the most frequent responses made by students, and, given one of these instances, the category was the most frequent response.

From the original seven categories, four were chosen that had instances most consistent with the Battig and Montague (1969) norms. The categories and their instances are as follows: bird-robin, sparrow; fish-trout, goldfish; flower-rose, daisy; tree-oak, apple tree. All instances were ranked within the top 10 in the Battig and Montague norms. It is understandable that the local and the Battig and Montague norms should differ slightly, since category structure varies with geography.

The low-typicality members of a category were chosen from the Battig and Montague (1969) norms with the restriction that the frequency with which subjects selected an instance first be no less than 10 and no greater than 20 , with an average ranking of 36.5. The instances chosen are as follows: bird-ostrich, stork; fish-sardine, piranha; flower-marigold, camellia; tree-magnolia, cypress.

Structure of the statements (high-typicality instance and lowtypicality instance) was orthogonally contrasted with type of statement (general and particular) to yield four experimental conditions. Subjects were given a booklet composed of 16 problems ( 8 affirmative and 8 negative) constructed in the following manner. First, categories were randomly assigned to statements with the restriction that an animate category in one statement be contrasted with an inanimate category in the other statement. Second, each pair appeared in four arrangements determined by which category relation served as the generality or the particular fact and whether the relations were affirmatively or negatively expressed. Third, order of sentences was counterbalanced so that the general and particular statements occurred as the first statement on the page an equal number of times.

At the beginning of an experimental session, instructions were read aloud by the experimenter while the subjects followed with their own copies of the instructions. A sample problem was presented and the two possible solutions were demonstrated. The subjects were then told to proceed throughout the problems at their own rates, crossing out all statements that led to a logical contradiction with the assumption. The subjects were further asked to work on each problem in the order presented in the booklet (one problem per page). Any questions regarding the logical ambiguity of the solutions were answered by instructing the subjects to choose the "best" alternative. 


\section{RESULTS}

Each subject received a score for the number of times a generality was assigned "true." This was done separately for affirmative and negative problems. The data are presented in Table 1, which reveals two basic findings. First, the decisions reached in the present task replicate the overall findings of Revlis et al. (1971): (1) Generalities were assigned "true" more often than would be expected by chance alone (Wilcoxon matchedpairs signed-ranks test, $p<.05$ ), (2) affirmative generalities were assigned "true" more often than were negative ones $[F(1,92)=17.8, p<.01]$. Second, no significant effect of typicality of the category instances was found. In contrast with previous findings, negative generalities were assigned "true" more often than chance only in the case where the general relations were highly typical and their contrasting facts were of low typicality. The present data suggest that the findings of Revlis et al. (1971) are not due to a confounding of semantic structure and statement generality: No difference in overall acceptance of generalities was found between the groups where generality and fact were of equally high typicality and where the generality was highly typical but the fact was of low typicality.

\section{DISCUSSION}

It is clear from these data that semantic structure as defined here is not a sufficient criterion for acceptance of statements in belief-contravening problems. When generalities affirm a relation, they are readily accepted as defining the hypothetical domain. The addition of category structure makes only a marginal contribution to prevaricative judgments. Of course, it is possible that the acceptability of the generalities in the present study represents a maximum level, so that any increase due to the addition of within-categories structure would not be observed.

In contrast, there is a discernible semantic structure effect for negatives that merits an explanation, however tentatively advanced. When presented with negative generalities, subjects still show a slight overall preference for them (Wilcoxon test, $\mathrm{p}<.05)$. This preference is considerably less than is shown for affirmative generalities. Because the negatives provide only weak structuring the judgments are difficult and may be of low confidence. We conjecture that subjects more deeply analyze the negatives (in contrast with affirmatives) to discern some relevant components that will facilitate their judgments. Semantic elements present in category structure may provide the necessary distinguishing features.

The two-stage model presented earlier claims that subjects attend primarily to the generalities to extract relevant elements. When negatives have high structure, subjects then analyze the

Table 1

Percent Acceptance of Generalities with Different Category Structure

\begin{tabular}{clll}
\hline $\begin{array}{c}\text { Structure of } \\
\text { Generalities }\end{array}$ & Polarity & \multicolumn{2}{c}{$\begin{array}{c}\text { Structure of } \\
\text { Particulars }\end{array}$} \\
\hline \multirow{2}{*}{ High } & Affirmative & 74.1 & 76.8 \\
& Negative & 56.1 & 72.3 \\
Low & Affirmative & 67.3 & 70.8 \\
& Negative & 54.6 & 58.9 \\
\hline
\end{tabular}

contrasting facts to determine whether the attention to such features is warranted. In the condition where both generality and fact are of high typicality or both are of low typicality, the features are nondistinguishing and are, therefore, not a part of the judgments. In the low-generality/high-fact condition, subjects do not attend to the increased structure that is present in the particular facts, since the semantic structuring does not exist initially in their analyses of the generalities. However, in the high-generality/low-particular condition, the features are pertinent because of their ability to distinguish between the two statements and lend support to the incipient bias toward generalities. This analysis correctly predicts that increased acceptability of negative generalities should be found only in the condition where the semantic structure increases confidence in the generality, but not in the fact: the high-generality/low-fact condition.

The foregoing hypothesis is only tentatively advanced as an explanation for the reasoning process on hypothetical inference tasks, but it strongly suggests the importance of analyzing confidence and decision-time measures.

Related to the foregoing is the study by Revlis and Hayes (1972) that examined the importance of certain sentential features for their ability to influence the acceptability of generalities in hypothetical reasoning. These features were the major ones developed by Goodman (1947) and Rescher (1964) for defining a scientific law. The authors found a polarity effect in all cells except one: that case where the generalities (including negative ones) fulfilled the definitions for lawfulness while the contrasting particular statements did not. In that condition, affirmative and negative generalities were equivalent in their attractiveness for organizing the hypothetical domain. The present findings show a similar pattern: the absence of a polarity effect in that condition where only the generalities possess maximum semantic structure. Perhaps "lawfulness" and semantic structure are related concepts that may be empirically investigated using belief-contravening problems.

\section{REFERENCES}

Battig, W. F., \& Montague, W. E. Category norms for verbal items in 56 categories. Journal of Experimental Psychology Monograph, 1969, 80(3, Part 2).

Goodman, N. The problem of counterfactual conditionals. In L. Linsky (Ed.), Semantics and the philosophy of language. Urbana: University of Illinois Press, 1947.

Hocketr, C. F. The problem of universals in language. In J. H. Greenberg (Ed.), Universals of language (2nd ed.). Cambridge: MIT Press, 1966.

Rescher, N. Hypothetical Reasoning. Amsterdam: NorthHolland, 1964.

Revlis, R. Prevarication: Reasoning from false assumptions. Memory \& Cognition, 1974, 2, 87-95.

Revlis, R., \& Hayes, J. R. The primacy of generalities in hypothetical reasoning. Cognitive Psychology, 1972, 3, 268-290.

Revlis, R., LIPKIN, S., \& HaYes, J. The importance of universal quantifiers in a hypothetical reasoning task. Journal of Verbal Learning and Verbal Behavior, 1971, 10, 86-91.

Rosch, E. On the internal structure of perceptual and semantic categories. In T. E. Moore (Ed.), Cognitive development and acquisition of language. New York: Academic Press, 1973.

Rosch, E., \& Mervis, C. B. Family resemblances: Studies in the internal structure of categories. Cognitive Psychology, 1975, 7, 573-605.

RyLE, G. The concept of mind. New York: Barnes \& Noble, 1949.

(Received for publication September 6, 1978.) 